\title{
Quasi-Relativistic Description of Hydrogen-Like Atoms
}

\author{
Luis Grave de Peralta \\ Department of Physics and Astronomy, Texas Tech University, Lubbock, TX, USA \\ Email: luis.grave-de-peralta@ttu.edu
}

How to cite this paper: de Peralta, L.G. (2020) Quasi-Relativistic Description of Hydrogen-Like Atoms. Journal of Modern Physics, 11, 788-802.

https://doi.org/10.4236/jmp.2020.116051

Received: May 7, 2020

Accepted: May 30, 2020

Published: June 2, 2020

Copyright $\odot 2020$ by author(s) and Scientific Research Publishing Inc. This work is licensed under the Creative Commons Attribution International License (CC BY 4.0).

http://creativecommons.org/licenses/by/4.0/

\begin{abstract}
Using a novel wave equation, which is Galileo invariant but can give precise results up to energies as high as $m c^{2}$, exact quasi-relativistic quantum mechanical solutions are found for the Hydrogen atom. It is shown that the exact solutions of the Grave de Peralta equation include the relativistic correction to the non-relativistic kinetic energies calculated using the Schrödinger equation.
\end{abstract}

\section{Keywords}

Quantum Mechanics, Schrödinger Equation, Relativistic Quantum

Mechanics, Klein-Gordon Equation, Hydrogen Atom

\section{Introduction}

Quantum mechanics triumphed when physicists learned to describe the quantum states of the electrons in the atoms by solving the Schrödinger equation [1] [2] [3] [4] [5]. However, the Schrödinger equation is not Lorentz invariant but Galilean invariant [6] [7]; therefore, a relativistic quantum mechanics cannot be based on the Schrödinger equation. A fully relativistic quantum theory requires to be funded on equations that are valid for any two observers moving respect to each other at constant velocity [8] [9]. In contrast, the Galilean invariance of the Schrödinger equation means that two such observers will only agree in the adequacy of the Schrödinger equation for describing the movement of a massive free quantum particle when the relative speed between the observers is much smaller than the speed of the light in the vacuum (c). In practice, this is not a terrible limitation of the Schrödinger equation because up to today humans have been only able to travel at speeds much smaller than $c$. This is one of the principal reasons why the Schrödinger equation is still relevant almost 100 years after 
its discovery. Moreover, there is another important limitation of the Schrödinger equation: it describes a particle in which linear momentum $(p)$ and kinetic energy $(K)$ are related by a classical relation that is not valid at relativistic speeds [6] [7] [8] [9] [10]. Nevertheless, wave mechanics triumphed when Schrödinger, using his equation, was able to reproduce the results previously obtained by Bohr for the energies of the bound states of the electron in the Hydrogen atom. This was possible because the electron in the Hydrogen atom moves at non-relativistic energies [1] [2] [3] [4] [5]. Rigorously, the number of particles may be not constant in a fully relativistic quantum theory [7] [8] [9]. This is because when the sum of the kinetic and the potential $(U)$ energy of a particle with mass $m$ equals the energy associate to the mass of the particle, i.e. $E=K+U=m c^{2}$, then a second particle with the same mass could be created from $E$. Consequently, the number of particles is constant when $E=K+U<m c^{2}$. This is what happens in atoms and molecules; thus, this explains why the results obtained using the Schrödinger equation are a good first approximation in chemistry applications [5]. In between the Galilean invariant Schrödinger equation and the fully relativistic quantum mechanics, there is a quasi-relativistic region where $E<m c^{2}$ but $E$ is so large that it is necessary to use an equation that describes a particle having a relativistic relation between $p$ and $K$. In this work, the use of the Grave de Peralta equation is explored [7] [11], which is a quasi-relativistic wave equation, for describing the bounded states of an electron in a Hydrogen-like atom with atomic number $Z$. It is shown that the energies calculated using the Grave de Peralta equation are in excellent correspondence with the sum of the energies calculated using the Schrödinger equation plus the relativistic Thomas correction [12]. This demonstrates both the correctness and the usefulness of the proposed approach at quasi-relativistic energies. In what follows, first, a brief summary of the Grave de Peralta equation and its basic properties is presented; then solutions of this equation are obtained for a central potential in general and for the Coulomb potential. Finally, the conclusions of this work are given in the last section.

\section{The Grave de Peralta Equation}

Formally, the one-dimensional (1D) Schrödinger equation for a free quantum particle with mass $m$ can be obtained from the classical relation between $K$ and $p$ for a free particle when its speed $(V)$ is much smaller than the $c$ [1] [2] [3] [4] [5]:

$$
K=\frac{p^{2}}{2 m}, \quad p=m V .
$$

Then, substituting $K$ and $p$ by the following energy and momentum quantum operators [1] [2] [3] [4]:

$$
\hat{E}=\hat{K}=i \hbar \frac{\partial}{\partial t}, \quad \hat{p}=-i \hbar \frac{\partial}{\partial x} .
$$


In Equation (2), $\hbar$ is the Plank constant ( $h$ ) divided by $2 \pi$, results [1] [2] [3] [4] [5]:

$$
i \hbar \frac{\partial}{\partial t} \psi_{\text {Sch }}(x, t)=-\frac{\hbar^{2}}{2 m} \frac{\partial^{2}}{\partial x^{2}} \psi_{\text {Sch }}(x, t) .
$$

However, Equation (1) does not give the correct relation between $K$ and $p$ when the particle moves at faster speeds. Correspondingly, the Schrödinger equation (Equation (3)) is not Lorentz invariant but Galileo invariant [6] [7]; thus, only should be used for particles moving slowly. At larger particle's speed, one should use the following well-known relativistic relations [10]:

$$
E^{2}-m^{2} c^{4}=p^{2} c^{2} \Leftrightarrow\left(E+m c^{2}\right)\left(E-m c^{2}\right)=p^{2} c^{2} .
$$

And:

$$
E=\gamma_{V} m c^{2}, p=\gamma_{V} m V .
$$

Here, $E=K+m c^{2}$ is the total relativistic energy of the free particle, and [10]:

$$
\gamma_{V}=\frac{1}{\sqrt{1-\frac{V^{2}}{c^{2}}}}
$$

One can then formally proceed as it is done for obtaining the 1D Schrödinger equation, and use Equation (2) for assigning the temporal partial derivative operator to $E$ in the first expression of Equation (4) [7] [8] [9]. In this way, one can formally obtain the 1D Klein-Gordon equation [8] [9]:

$$
\frac{1}{c^{2}} \frac{\partial^{2}}{\partial t^{2}} \psi_{K G}(x, t)=\frac{\partial^{2}}{\partial x^{2}} \psi_{K G}(x, t)-\frac{m^{2} c^{2}}{\hbar^{2}} \psi_{K G}(x, t) .
$$

The Klein-Gordon equation is Lorentz invariant and describes a free quantum particle with mass $m$ and spin-0 [8] [9]. In contrast to the Schrödinger equation, a second-order temporal derivative is present in Equation (7). This determines that Equation (7) has solutions with positive and negative energy values while Equation (3) has only solutions with positive energies, which is in correspondence with $K$ having only positive values in Equation (1) but $E$ having positive and negative values in Equation (4). The factor $\left(E+m c^{2}\right)$ is always different than 0 for $E>0$; consequently, Equation (4) and the following algebraic equation are equivalents for $E>0$ :

$$
\left(E-m c^{2}\right)=\frac{p^{2}}{\left(\gamma_{V}+1\right) m}
$$

Each member of Equation (8) is just a different expression of the relativistic kinetic energy of the particle [7]. Assigning the temporal partial derivative operator in Equation (2) to $E$ in Equation (8) results in the following differential equation [11]:

$$
i \hbar \frac{\partial}{\partial t} \psi_{K G+}(x, t)=-\frac{\hbar^{2}}{\left(\gamma_{V}+1\right) m} \frac{\partial^{2}}{\partial x^{2}} \psi_{K G+}(x, t)+m c^{2} \psi_{K G+}(x, t) .
$$

A simple substitution in Equation (7) and Equation (9) shows that the follow- 
ing plane wave is a solution of both equations for $E>0$ :

$$
\psi_{K G+}(x, t)=\mathrm{e}^{\frac{i}{\hbar}(p x-E t)} .
$$

The plane wave $\psi_{K G+}$ has an unphysical phase velocity equal to $c^{2} / V>c$ [7] [11]. However, one can look for a solution of Equation (9) of the following form:

$$
\psi(x, t)=\psi_{K G+} \mathrm{e}^{i w_{m} t}, w_{m}=\frac{m c^{2}}{\hbar} .
$$

Such that $\psi$ has a phase velocity smaller than $c$ [7] [11]; thus:

$$
\psi(x, t)=\mathrm{e}^{\frac{i}{\hbar}(p x-K t)} .
$$

Substituting $\psi$ given by Equation (11) in Equation (9) results in the 1D Grave de Peralta equation [7] [11]:

$$
i \hbar \frac{\partial}{\partial t} \psi(x, t)=-\frac{\hbar^{2}}{\left(\gamma_{V}+1\right) m} \frac{\partial^{2}}{\partial x^{2}} \psi(x, t) .
$$

Equation (13) clearly coincides with the Schrödinger equation at low particle's speeds. Moreover, a positive probability density can be defined for the solutions of Equation (13) by analogy of how it is defined for the solutions of the Schrödinger equation and, like the Schrödinger equation, Equation (13) is Galilean invariant for observers traveling at low speeds respect to each other [7]. Despite this, Equation (13) can be used for obtaining precise solutions of very interesting quantum problems at quasi-relativistic energies [7] [11], where a particle moves at so large speeds that it is necessary to use the correct relativistic relation between $p$ and $K$, but where the particle should not be moving too fast so that the number of particles remains constant. When the particle moves through a $1 \mathrm{D}$ piecewise constant potential $U(x)$, Equation (13) should be generalized in the following way [11]:

$$
i \hbar \frac{\partial}{\partial t} \psi(x, t)=-\frac{\hbar^{2}}{\left[\gamma_{V}(x)+1\right] m} \frac{\partial^{2}}{\partial x^{2}} \psi(x, t)+U(x) \psi(x, t) .
$$

Often, one looks for solutions of Equation (14) corresponding to a constant value of the energy $E=K+U$, where $E$ is not the total relativistic energy of the particle $(E)$ but $E=E-m c^{2}$. At quasi-relativistic energies, the number of particles is constant; therefore, $E$ is constant whenever $E$ is constant. For a $1 \mathrm{D}$ piecewise constant potential $E, K, \gamma_{V}$, and $V^{2}$ are constants in each $x$-region where $U$ is constant. In contrast to $E$, however, $K, \gamma_{V}$ and $V^{2}$ have a discontinuity wherever $U(x)$ has one. Consequently, in Equation (14) $\gamma_{V}$ is a function of $x$ because, in general, the square of the particle's speed $\left(V^{2}\right)$ depends on the position [11]. Nevertheless, for 1D piecewise constant potentials, one can look for a solution of Equation (14) with the following form in each of the regions where $K, \gamma_{v}$ and $V^{2}$ are constants [1] [2] [3] [4] [11]:

$$
\psi(x, t)=X_{K}(x) \mathrm{e}^{-\frac{i}{\hbar} E t}, E=K+U
$$


$X_{K}$ is a solution of the following equation [1] [2] [3] [4] [11]:

$$
\frac{\mathrm{d}^{2}}{\mathrm{~d} x^{2}} X_{K}(x)+\kappa^{2} X_{K}(x)=0, \kappa=\frac{p}{\hbar} \text {. }
$$

And [11]:

$$
\kappa=\frac{p}{\hbar}=\frac{1}{\hbar} \sqrt{\left(\gamma_{V}+1\right) m K}=\frac{1}{\hbar} \sqrt{\left(\gamma_{V}+1\right) m(E-U)} .
$$

Consequently, $\kappa$ and $X_{K}$ are not determined by the values of $E$ but by the values of $K=E-U$. Once the allowed values of $\kappa$ are determined from Equation (16) and the boundary conditions, the allowed values of the relativistic kinetic energy of the particle $K=E-U$ are given by:

$$
K=\frac{\hbar^{2} \kappa^{2}}{\left(\gamma_{V}+1\right) m}
$$

As expected, when $\gamma_{V} \sim 1$, Equation (18) gives the non-relativistic values of the particle's energies at low speeds, $K \sim \hbar^{2} \kappa^{2} /(2 m)$ [1] [2] [3] [4]. Moreover, from Equation (18) and the relativistic equation, $K=\left(\gamma_{V}-1\right) m c^{2}$, follows that [11]:

$$
\gamma_{V}^{2}=1+\left(\frac{\lambda_{C}}{\lambda}\right)^{2}, \lambda_{C}=\frac{h}{m c}, \lambda=\frac{2 \pi}{\kappa} .
$$

In Equation (19), $\lambda_{C}$ is the Compton wavelength associate to the mass of the particle [8] [10], and $\lambda$ is the De Broglie wavelength of the wavefunction given by Equation (7) and Equation (10) [1] [2] [3] [4]. Substituting Equation (19) in Equation (18) allows obtaining an analytical expression of the precise quasi-relativistic kinetic energy of the particle:

$$
K=\frac{\hbar^{2} \kappa^{2}}{\left[1+\sqrt{1+\left(\frac{\lambda_{C}}{\lambda}\right)^{2}}\right] m} .
$$

As expected, Equation (20) match the non-relativistic expression of the particle's kinetic energy when $p=h / \lambda$ is very small because $\lambda \gg \lambda_{C}$. However, in each region where the value of $U$ is constant, the values of $K$ and then $E=K+U$ calculated using Equation (20) are smaller than the ones calculated using the Schrödinger equation.

\section{Movement in a Central Potential}

A quantum state of a particle with mass $m$ moving at quasi-relativistic energies in a central potential, $U(r)$, is a solution of the following 3D Grave de Peralta equation [7]:

$$
i \hbar \frac{\partial}{\partial t} \psi(\boldsymbol{r}, t)=-\frac{\hbar^{2}}{\left[\gamma_{V}(r)+1\right] m} \nabla^{2} \psi(\boldsymbol{r}, t)+U(r) \psi(\boldsymbol{r}, t) .
$$

In Equation (21), $\gamma_{V}$ and $V^{2}$ depend only on the radial variable $(r)$ because the 
potential is a central potential. In spherical coordinates, the Laplacian operator in Equation (21) is given by the following expression [1]:

$$
\nabla^{2} \psi=\frac{1}{r} \frac{\partial^{2}}{\partial r^{2}}(r \psi)+\frac{1}{r^{2}} \nabla_{\theta, \varphi}^{2} \psi
$$

In Equation (22):

$$
\nabla_{\theta, \varphi}^{2}=\frac{1}{\sin \theta} \frac{\partial}{\partial \theta}\left(\sin \theta \frac{\partial}{\partial \theta}\right)+\frac{1}{\sin ^{2} \theta} \frac{\partial^{2}}{\partial \varphi^{2}} .
$$

Using Equation (22) and Equation (23) allows for rewriting Equation (21) in the following way [7]:

$$
i \hbar \frac{\partial}{\partial t} \psi=-\frac{\hbar^{2}}{\left[\gamma_{V}(r)+1\right] m r} \frac{\partial^{2}}{\partial r^{2}}(r \psi)-\frac{\hbar^{2}}{\left[\gamma_{V}(r)+1\right] m r^{2}} \nabla_{\theta, \varphi}^{2} \psi+U(r) \psi .
$$

The second term of the right size of Equation (24) corresponds to the rotational energy of the particle. For a quantum rotor, which describes a particle moving in a sphere, $r$ is constant [2] [5]. This allows for simplifying Equation (24) in the following way [7]:

$$
i \hbar \frac{\partial}{\partial t} \psi(\theta, \varphi)=-\frac{\hbar^{2}}{\left(\gamma_{V}+1\right) m r^{2}} \nabla_{\theta, \varphi}^{2} \psi(\theta, \varphi) .
$$

The explicit absence of a potential in Equation (25) determines that it has solutions with constant values of $E, K, \gamma_{V}$ and $V^{2}$ [7]. However, one should expect to have solution of Equation (24) with constant values of $E$, but all $K, \gamma_{V}$ and $V^{2}$ depending on $r$. Looking for a solution of Equation (24) as in Ref. [1]:

$$
\psi(r, \theta, \varphi, t)=R(r) \Omega(\theta, \varphi) \mathrm{e}^{\frac{i}{\hbar} E t} .
$$

Results:

$$
\nabla_{\theta, \varphi}^{2} \Omega(\theta, \varphi)=\eta \Omega(\theta, \varphi)
$$

And:

$$
\frac{1}{r} \frac{\mathrm{d}^{2}}{\mathrm{~d} r^{2}}(r R)+\frac{\left[\gamma_{V}(r)+1\right] m r^{2}}{\hbar^{2}}[E-U(r)] R=-\eta \frac{R}{r^{2}} .
$$

Equation (27) is the well-known equation for the spherical harmonic functions [1] [2] [3] [4] [5], which solutions are:

$$
\Omega_{l, m}(\theta, \varphi)=Y_{l}^{(m)}(\theta, \varphi) ; \eta=l(l+1) ; l=0,1,2, \cdots ; m=-l,-l+1, \cdots, 0,1, \cdots, l .
$$

Here, $Y_{l}^{(m)}$ are the spherical harmonic functions [1] [2] [3] [4] [5]. Substituting $\eta$ given by Equation (29) in Equation (28) and looking for a solution of the form $R(r)=\chi(r) / r$ as in Ref. [4], then results the following equation:

$$
\frac{\mathrm{d}^{2}}{\mathrm{~d} r^{2}} \chi(r)+\frac{\left[\gamma_{V}(r)+1\right] m}{\hbar^{2}}\left[E_{c}-W(r)\right] \chi(r)=0 .
$$

In Equation (30):

$$
W(r)=\left[U(r)+\frac{\hbar^{2}}{\left[\gamma_{V}(r)+1\right] m} \frac{l(l+1)}{r^{2}}\right]
$$


The radial equation, Equation (30), is then formally identical to Equation (16) with:

$$
\kappa(r)=\frac{p(r)}{\hbar}=\frac{1}{\hbar} \sqrt{\left[\gamma_{V}(r)+1\right] m K(r)}=\frac{1}{\hbar} \sqrt{\left[\gamma_{V}(r)+1\right] m[E-W(r)]} .
$$

At low particle speeds, $\gamma_{V}(r) \sim 1$, thus Equation (30) coincide with the radial equation that can be obtained when solving the same problem using the Schrödinger equation [1] [2] [3] [4] [5]. However, at quasi-relativistic energies, $\gamma_{V}$ depends on $r$, therefore, in general, the solutions of Equation (30) are different than the solutions of the radial equation for the Schrödinger equation. A notable exception is the infinite spherical well problem for $I=0$ where $U(r)$ is given by the following expression [4]:

$$
U(r)= \begin{cases}0, & r<r_{o} \\ U_{o} \rightarrow+\infty, & r \geq r_{o}\end{cases}
$$

In this case $W(r) \equiv 0$; thus $K, \gamma_{V}$, and $V^{2}$ are constant inside the well. Equation (30) can then be solved as it is done for the Schrödinger equation. Consequently [4]:

$$
E_{n}=K_{n}=n^{2} \frac{h^{2}}{\left(\gamma_{V}+1\right) m D_{o}^{2}} .
$$

In Equation (34), $D_{o}=2 r_{o}$ and $n$ is a positive integer number. From Equation (34) and the relativistic equation $K=\left(\gamma_{V}-1\right) m c^{2}$ follows that:

$$
\gamma_{V}^{2}=1+n^{2}\left(\frac{\lambda_{C}}{D_{o}}\right)^{2}, \lambda_{C}=\frac{h}{m c} .
$$

Substituting $\gamma_{V}$ given by Equation (35) in Equation (34) results:

$$
E_{n}=\frac{n^{2}}{\left[1+\sqrt{1+\left(\frac{n \lambda_{C}}{D_{o}}\right)^{2}}\right]}\left(\frac{\lambda_{C}}{D_{o}}\right)^{2} m c^{2} .
$$

As expected, $\gamma_{V} \sim 1$ when $n=1$ and $D_{o} \gg \lambda_{C}$; thus, Equation (36) coincides with the energies of the infinite spherical well calculated using the Schrödinger equation [4]. In contrast, when the diameter of the well is close to $\lambda_{C}$ the minimum particle energy is quasi-relativistic; therefore, Equation (36) must be used. For instance, $\gamma_{V}^{2}=2, V \sim 0.7 c$, and $K \sim 0.4 m c^{2}$ when Equation (35) is evaluated for $n=1$ and $D_{o}=\lambda_{C}$. However, $\gamma_{V}^{2}=5$ and $K \sim 1.2 m c^{2}$ when $n=1$ and $D_{o}=$ $\lambda_{C}$ 2. The number of particles may not be constant at these energies. Consequently, the Grave de Peralta equation establishes a fundamental connection between quantum mechanics and especial theory of relativity: no single particle with mass can be confined in a volume much smaller than $1 / 8 \lambda_{C}^{3}$ because when this occurs, $K>m c^{2}$ and the number of particles may not be constant anymore; therefore, a single point-particle with mass cannot exist. Point-particles with mass can only exist in fully relativistic quantum field theories where the number of particles is not constant. This is true for an electron, a quark, and probably 
may also be true for a black hole and the whole universe at the beginning of the Big Bang. This is consistent, for instance, with the confinement of an electron in the Hydrogen atom because for an electron $\lambda_{C} \sim 2.4 \times 10^{-3} \mathrm{~nm}$, which is $\sim 20$ times smaller than the Bohr radius of the Hydrogen atom, $r_{B} \sim 5.3 \times 10^{-2} \mathrm{~nm}[1]$ [2] [3] [4] [5]. It should be noted that strictly speaking, the problem corresponding to the potential defined by Equation (33) is a relativistic problem because $|\Delta U|=U_{o} \gg m c^{2}$ and thus the number of particles may not be constant. Nevertheless, the non-relativistic and quasi-relativistic infinite well problems could be considered approximations to the problem of a quantum particle absolutely trapped in a finite region. This is because for obtaining Equation (34) and Equation (36) the infinitude of the potential is only used for arguing that $\chi(r)$ should be null everywhere except inside of the well, thus assigning null boundary conditions to Equation (30).

\section{Hydrogen-Like Atoms}

In the Hydrogen atom or in highly ionized atoms with a single electron, $U(r)$ is the Coulomb potential [1] [2] [3] [4] [5]:

$$
U(r)=U_{C}(r)=-\frac{e^{2}}{4 \pi \varepsilon_{o}} \frac{Z}{r} .
$$

Here, $e$ is the electron charge, $Z$ is the atomic number, and $\varepsilon_{o}$ is the electric permittivity of vacuum. Therefore, the radial equation corresponding to the quasi-relativistic states of the electron in a hydrogen-like atom with a nucleus of mass $m_{n}$ is given by the Equation (30) with the electron mass, $m_{e}$, substituted by the reduced mass of the electron, $\mu=\left(m_{e} m_{n}\right) /\left(m_{e}+m_{n}\right)$, i.e.:

$$
\frac{\mathrm{d}^{2}}{\mathrm{~d} r^{2}} \chi(r)+\frac{\left[\gamma_{V}(r)+1\right] \mu}{\hbar^{2}}\left[E_{c}-W_{C}(r)\right] \chi(r)=0 .
$$

In Equation (38):

$$
W_{C}(r)=\left[U_{C}(r)+\frac{\hbar^{2}}{\left[\gamma_{V}(r)+1\right] \mu} \frac{l(l+1)}{r^{2}}\right] .
$$

As expected, when the electron moves slowly $(V \ll c)$ then $\gamma_{V} \sim 1$; therefore, Equation (38) reduces to the radial equation of a hydrogen-like atom obtained using the Schrödinger equation [4]. Using Equation (5), it is possible to eliminate $\gamma_{V}$ from Equation (38) and Equation (39) by making:

$$
\frac{\left[\gamma_{V}(r)+1\right] \mu}{\hbar^{2}}=\frac{K+2 \mu c^{2}}{c^{2} \hbar^{2}}=\frac{\left[E-U_{C}(r)\right]+2 \mu c^{2}}{c^{2} \hbar^{2}} .
$$

Using Equation (40) then allows for rewriting Equation (38) in the following way:

$$
\begin{aligned}
& \left\{-\frac{\hbar^{2}}{2 \mu} \frac{\mathrm{d}^{2}}{\mathrm{~d} r^{2}} \chi(r)-\left[E-U_{C}(r)\right] \chi(r)+\frac{\hbar^{2}}{2 \mu} \frac{l(l+1)}{r^{2}} \chi(r)\right\} \\
& -\frac{1}{2 \mu c^{2}}\left[E-U_{C}(r)\right]^{2} \chi(r)=0 .
\end{aligned}
$$


The term between braces in Equation (41) coincides with the radial equation that should be solved when using the Schrödinger equation [4]. The last term of Equation (41) can be disregarded when $K \ll \mu c^{2}$; therefore, the last term is a quasi-relativistic correction to the non-relativistic radial equation. Proceeding like it is done when solving the non-relativistic radial equation, one can introduce [4]:

$$
\zeta=\frac{1}{\hbar} \sqrt{-2 \mu E} .
$$

For bound states, $E<0$; therefore, $\zeta$ is real. Using Equation (42) allows for rewriting Equation (41) in the following way:

$$
\begin{aligned}
\frac{1}{\zeta^{2}} \frac{\mathrm{d}^{2}}{\mathrm{~d} r^{2}} \chi(r)= & \left\{\left[1-\frac{\mu e^{2}}{2 \pi \varepsilon_{o} \hbar^{2} \zeta} \frac{Z}{(\zeta r)}+\frac{l(l+1)}{(\zeta r)^{2}}\right]\right. \\
& \left.-\left[\frac{\alpha^{2} Z^{2}}{(\zeta r)^{2}}-\frac{\hbar \zeta}{\mu c} \frac{\alpha Z}{(\zeta r)}+\left(\frac{\hbar}{2 \mu c}\right)^{2} \zeta^{2}\right]\right\} \chi(r) .
\end{aligned}
$$

where $\alpha$ is the fine-structure constant [8] [9] [12]:

$$
\alpha=\frac{1}{4 \pi \varepsilon_{o}} \frac{e^{2}}{\hbar c} \sim 1 / 137 \text {. }
$$

It is convenient to rewrite Equation (43) as:

$$
\begin{aligned}
\frac{1}{\zeta^{2}} \frac{\mathrm{d}^{2}}{\mathrm{~d} r^{2}} \chi(r)= & \left\{\left[1-\left(\frac{\hbar \zeta}{2 \mu c}\right)^{2}\right]-\left[\left(\frac{\mu e^{2}}{2 \pi \varepsilon_{o} \hbar^{2} \zeta}-\alpha \frac{\hbar \zeta}{\mu c}\right) \frac{Z}{(\zeta r)}\right]\right. \\
& \left.+\frac{l(l+1)-\alpha^{2} Z^{2}}{(\zeta r)^{2}}\right\} \chi(r) .
\end{aligned}
$$

So that introducing the new variables:

$$
\rho \equiv \zeta r, \quad \rho_{o} \equiv\left(\frac{\mu e^{2}}{2 \pi \varepsilon_{o} \hbar^{2} \zeta}-\alpha \frac{\hbar \zeta}{\mu c}\right) Z, \rho_{1} \equiv\left[1-\left(\frac{\hbar \zeta}{2 \mu c}\right)^{2}\right] .
$$

Allows for rewriting Equation (45) in the following way:

$$
\frac{\mathrm{d}^{2}}{\mathrm{~d} \rho^{2}} \chi(\rho)=\left[\rho_{1}-\frac{\rho_{o}}{\rho}+\frac{l(l+1)-\alpha^{2} Z^{2}}{\rho^{2}}\right] \chi(\rho) .
$$

If the electron was free and moving slowly with kinetic energy $K=E$, then its linear momentum would be $\hbar \zeta \ll \mu c$. In this limit, one can approximate Equation (46) in the following way [3] [4]:

$$
\rho \equiv \zeta r, \rho_{o} \sim \frac{\mu e^{2} Z}{2 \pi \varepsilon_{o} \hbar^{2} \zeta}, \rho_{1} \sim 1 .
$$

Using Equation (48), and considering that for the Hydrogen atom $\alpha^{2} Z^{2} \ll 1$, allows for approximating Equation (47) in the following way [3] [4]:

$$
\frac{\mathrm{d}^{2}}{\mathrm{~d} \rho^{2}} \chi(\rho)=\left[1-\frac{\rho_{o}}{\rho}+\frac{l(l+1)}{\rho^{2}}\right] \chi(\rho) \text {. }
$$


which is the equation that is solved for the Hydrogen atom when using the Schrödinger equation [4]. Consequently, when solving the Schrödinger equation can be found that [4]:

$$
\rho_{o}=2 n, n=1,2,3, \cdots
$$

From Equation (50), Equation (48), and Equation (42) then follows for $Z=1$ the following well-known result [2] [3] [4]:

$$
E_{n, S c h}=-\left[\frac{\mu}{2 \hbar^{2}}\left(\frac{e^{2}}{4 \pi \varepsilon_{o}}\right)^{2}\right] \frac{1}{n^{2}} .
$$

However, each of the three terms in the right side of Equation (47) contains a different quasi-relativistic correction to the radial equation of hydrogen-like atoms. Nevertheless, one can try to solve the quasi-relativistic Equation (47) as Equation (49) is solved [4]. When $\rho \rightarrow \infty$, the constant term in the brackets in Equation (47) dominates, so (approximately):

$$
\frac{\mathrm{d}^{2}}{\mathrm{~d} \rho^{2}} \chi(\rho)=\rho_{1} \chi(\rho)
$$

which general solution is:

$$
\chi(\rho)=A \mathrm{e}^{-\sqrt{\rho_{1}} \rho}+B \mathrm{e}^{\sqrt{\rho_{1}} \rho} .
$$

But $\rho_{1}>0$; therefore, $B$ must be null, so for large $\rho$ :

$$
\chi(\rho) \sim A \mathrm{e}^{-\sqrt{\rho_{1}} \rho} .
$$

On the other hand, when $\rho \rightarrow 0$ the centrifugal term dominates; approximately, then:

$$
\frac{\mathrm{d}^{2}}{\mathrm{~d} \rho^{2}} \chi(\rho)=\frac{l(l+1)-\alpha^{2} Z^{2}}{\rho^{2}} \chi(\rho) .
$$

Which general solution is:

$$
\chi(\rho)=C \rho^{\frac{1}{2}\left[1+\sqrt{(1+2 l)^{2}-4 \alpha^{2} Z^{2}}\right]}+D \rho^{\frac{1}{2}\left[1-\sqrt{(1+2 l)^{2}-4 \alpha^{2} Z^{2}}\right]} .
$$

Therefore, $D$ must be null, so for small $\rho$ :

$$
\chi(\rho) \sim C \rho^{\frac{1}{2}\left[1+\sqrt{(1+2 l)^{2}-4 \alpha^{2} Z^{2}}\right]} .
$$

As expected, if the quasi-relativistic corrections are very small, then Equation (54) and Equation (57) reduces to the ones obtained when using the Schrödinger equation [4]. After knowing the asymptotic behavior of $\chi(\rho)$, one can look for a solution of Equation (47) as [4]:

$$
\chi(\rho) \equiv \tau(\rho) \rho^{\frac{1}{2}\left[1+\sqrt{(1+2 l)^{2}-4 \alpha^{2} Z^{2}}\right]} \mathrm{e}^{-\sqrt{\rho_{1} \rho}} .
$$

From Equation (58) and Equation (47) then follow that $\tau(\rho)$ is a solution of the following equation: 


$$
\begin{aligned}
& \rho \frac{\mathrm{d}^{2}}{\mathrm{~d} \rho^{2}} \tau(\rho)+\left[1-\left(2 \sqrt{\rho_{1}}\right) \rho+\sqrt{(1+2 l)^{2}-4 \alpha^{2} Z^{2}}\right] \frac{\mathrm{d}}{\mathrm{d} \rho} \tau(\rho) \\
& +\left[\rho_{o}-\sqrt{\rho_{1}}\left(1+\sqrt{(1+2 l)^{2}-4 \alpha^{2} Z^{2}}\right)\right] \tau(\rho)=0 .
\end{aligned}
$$

Again, as expected, if the quasi-relativistic corrections are very small, then Equation (59) reduces to the one obtained when using the Schrödinger equation [4]. Finally, assuming that $\tau(\rho)$ can expressed as a finite power series in $\rho$ $[4]$ :

$$
\tau(\rho)=\sum_{j=0}^{j_{\max }} a_{j} \rho^{j}
$$

And substituting Equation (60) in Equation (59) results:

$$
a_{j+1}=\frac{\sqrt{\rho_{1}}\left[2 j+\left(1+\sqrt{(1+2 l)^{2}-4 \alpha^{2} Z^{2}}\right)\right]-\rho_{o}}{(j+1)\left[j+\left(1+\sqrt{(1+2 l)^{2}-4 \alpha^{2} Z^{2}}\right)\right]} a_{j} .
$$

Evaluating Equation (61) for $j=j_{\max }$ and making $a_{j_{\max }+1}=0$, results:

$$
\frac{\rho_{o}}{\sqrt{\rho_{1}}}=\left[2 j+\left(1+\sqrt{(1+2 l)^{2}-4 \alpha^{2} Z^{2}}\right)\right] \text {. }
$$

As expected, if the quasi-relativistic corrections are very small, then Equation (62) reduces to Equation (50) with $n=j+l+1$ [4]. Nevertheless, one can rewrite Equation (62) in the following way:

$$
\rho_{o}=[2 n+\Delta(l, Z)] \sqrt{\rho_{1}} \text {. }
$$

In Equation (63):

$$
\Delta(l, Z)=\left[\left(1+\sqrt{(1+2 l)^{2}-4 \alpha^{2} Z^{2}}\right)-2(l+1)\right] .
$$

Substituting $\rho_{o}$ and $\rho_{1}$ given by Equation (46) in Equation (63), solving the resulting equation for $\zeta$, and using Equation (42) allows for obtaining an exact analytical expression for $E$, which now depends not only on the principal quantum number $n$, but also on the angular quantum number $l$, and $Z$. For instance, assuming that the quasi-relativistic corrections included in $\rho_{o}$ and $\rho_{1}$ do not need to be accounting for because they are too small, the effect of the quasi-relativistic correction included in the centrifugal term in Equation (63) is quantified by the following equation:

$$
E_{n, l}=-\left[\frac{\mu}{2 \hbar^{2}}\left(\frac{e^{2}}{2 \pi \varepsilon_{o}}\right)^{2}\right] \frac{Z^{2}}{[2 n+\Delta(l, Z)]^{2}} .
$$

As expected, if $\alpha$ was null and $Z=1$, then Equation (65) would be identical to Equation (51). However, $\Delta(l, Z)<0$ and $|\Delta(l, Z)|$ increases when $Z$ increases. Therefore, for $n>1$ and $l>0$, the degeneration of $E_{n}$ given by Equation (51) is broken by the quasi-relativistic correction $\Delta(l, Z)$. This effect is more pronounced for heavy elements. In addition, as shown in Figure $1,|\Delta(l, Z)|$ decreases when $l$ increases; therefore, $E_{n, l} \rightarrow E_{n}$ when $l$ is large. Figure 1 shows a 


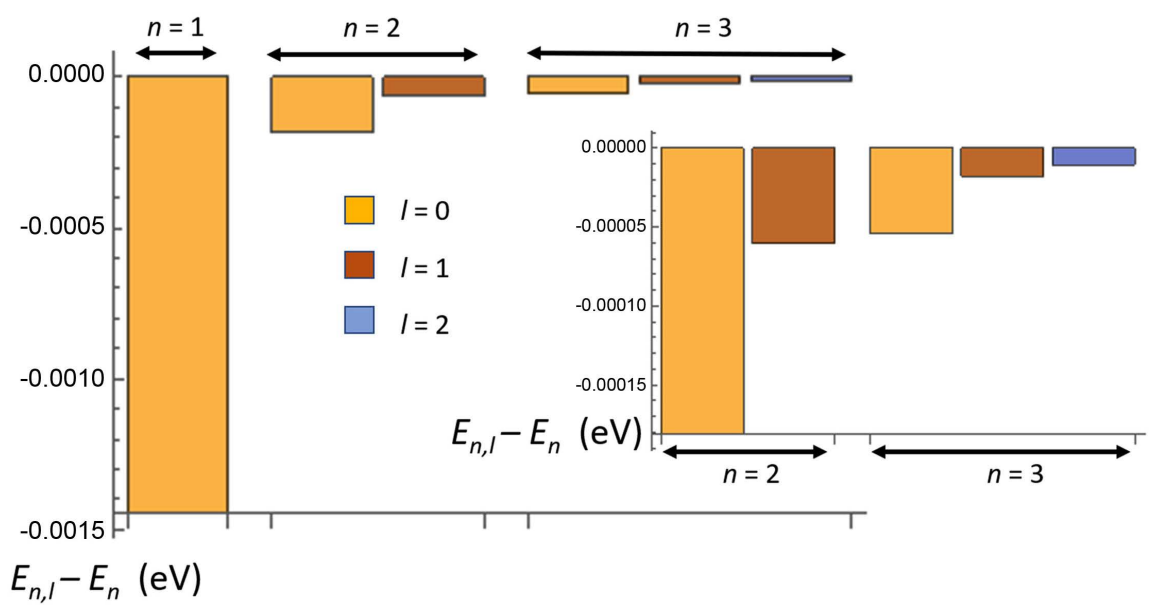

Figure 1. Schematic of the values of $E_{n, l}-E_{n}$ (in eV), which are calculated for $Z=1$ and $n$ $=1,2,3$ using Equation (65) and Equation (51), respectively. The inset at the right permits to appreciate the details corresponding to the energy levels $n=2$ and 3 .

schematic of the calculated values of $E_{n, l}-E_{c n}$ in $\mathrm{eV}$, where $E_{n, l}$ and $E_{n}$ were evaluated using Equation (65) with $Z=1$ and Equation (51), respectively. In all cases, stabilizing negative quasi-relativistic corrections to the non-relativistic energies were obtained. This is because the negative contribution of $-a^{2} Z^{2}$ in the numerator of the centrifugal term in Equation (47).

In columns 2 and 3 in Table 1 are reported the calculated values of $E_{n, l}(\mathrm{in} \mathrm{eV})$ that were calculated using Equation (65) and Equation (63), respectively. The difference between the approximated values (Equation (65)) and the exact values (Equation (63)) of $E_{n, l}$ are $\sim 0.01 \mathrm{meV}$; thus smaller than the exact values of $E_{n, l=0}-E_{n}$ reported in the third column of Table 1. Therefore, Figure 1 also represents a good schematic of the exact values of $E_{n, l}-E_{n}$; i.e. calculated using Equation (63) and Equation (51). There is an excellent correspondence between the exact values of $E_{n, l}-E_{n}$ reported in the third column of Table 1, and previously reported values of the relativistic correction to $E_{n}$ [4] [12]. This suggests that the solutions of Equation (63) are approximately equal to [4] [12]:

$$
E_{n, l} \sim E_{t n, S c h} Z^{2}\left\{1-\frac{\alpha^{2}}{n^{2}}\left[\frac{3}{4}-\frac{n}{l+\frac{1}{2}}\right]\right\} .
$$

where $E_{n, l s c h}$ given by Equation (51) correspond to the Hydrogen energies calculated using the Schrödinger equation. In correspondence with this, one can show that the values of $E_{n, l}$ calculated using Equation (65) are approximately equal to:

$$
E_{n, l} \sim E_{n, S c h} Z^{2}\left(1+\frac{2 \alpha^{2}}{n(2 l+1)}\right) .
$$

Indeed, Equation (65) can be rewritten as:

$$
E_{n, l}=-\mu c^{2} \alpha^{2} Z^{2} \frac{1}{[2 n+\Delta(l, Z)]^{2}} .
$$


Table 1. First four columns: Calculated values of $E_{n, l}$ (in eV) and $E_{n, l}-E_{n}$ (in meV). The last three columns report the calculated values of the energies (in $\mathrm{eV}$ ) and wavelengths (in $\mathrm{nm}$ ) corresponding to the Hydrogen's Lyman (second row) and average Balmer $\alpha$-line (sixth row), which were calculated using the exact quasi-relativistic values of $E_{n, l}$ reported in the third column.

\begin{tabular}{ccccccc}
\hline$(n, I)$ & Equation (65) & Equation (63) & $E_{n l}-E_{n}(\mathrm{meV})$ & $(n, I) \rightarrow(n, I)$ & $E_{n !}-E_{n, l}$ & $\lambda(\mathrm{nm})$ \\
\hline$(1,0)$ & -13.5997 & -13.5992 & -0.90526 & $(2,1) \rightarrow(1,0)$ & 10.1996 & 121.558 \\
$(2,0)$ & -3.39975 & -3.39972 & -0.147102 & $(3,1) \rightarrow(2,0)$ & 1.88879 & 656.422 \\
$(2,1)$ & -3.39963 & -3.3996 & -0.0264008 & $(3,0) \rightarrow(2,1)$ & 1.88863 & 656.477 \\
$(3,0)$ & -1.51097 & -1.51097 & -0.046938 & $(3,2) \rightarrow(2,1)$ & 1.88867 & 656.462 \\
$(3,1)$ & -1.51094 & -1.51093 & -0.0111749 & $(3) \rightarrow(2,1)$ & 1.88863 & 656.4695 \\
$(3,2)$ & -1.51093 & 1.51092 & -0.00402301 & & & \\
\hline
\end{tabular}

Then Equation (67) can be obtained from Equation (68) using the following approximated relations:

$$
\frac{1}{[2 n+\Delta(l, Z)]^{2}} \sim \frac{1}{(2 n)^{2}}\left[1-\frac{\Delta(l, Z)}{n}\right], \Delta(l, Z) \sim-\frac{2 \alpha^{2}}{2 l+1} .
$$

This is an important result: the quasi-relativist energies calculated using the Grave de Peralta equation corresponds to the sum of the non-relativistic energies calculated using the Schrödinger equation plus the relativistic corrections to the kinetic energy. Consequently, these energies do not include the Darwin energy term [12]. In addition, like the Schrödinger equation, Equation (21) and Equation (38) describe a charged particle with spin-0 moving in a Coulomb potential; therefore, no spin-orbit interaction is included in Equation (63) and Equation (65). It is well-known that both the Darwin and spin-orbit corrections are needed for a successful description of the Hydrogen spectrum [8] [9] [12]. Nevertheless, it is good to emphasize the improvement that can be obtained by using the Grave de Peralta equation in comparison to using the Schrödinger equation. The quasi-relativistic approximation to the Hydrogen spectral lines corresponding to the $\alpha$-lines of the Lyman and Balmer series can be estimated using the values of $E_{n, l}$ reported in the third column of Table 1 and the spectral rule $\Delta l= \pm 1$ [12]. The calculated values of $E_{n^{\prime}, l^{\prime}}-E_{n, l}$ (in eV), which correspond to all possible transitions between the states $(n, I)$ reported in the third column of Table 1, are reported in the sixth column of Table 1 . The corresponding wavelength values (in $\mathrm{nm}$ ) are reported in the last column of Table 1. Table 2 allows for making a breve comparison between the calculated values reported in the last two columns of Table 1 and previously reported experimental data [13] [14]. The doublet structure of the $\alpha$-Lyman Hydrogen line cannot be explained without the spin-orbit interaction because only the transition $(2,1)$ to $(1,0)$ satisfices the spectral rule $\Delta l= \pm 1$. However, the existence of the doublet fine-structure of the Balmer's $\alpha$-line could be calculated as corresponding to the $\lambda_{1}=656.422 \mathrm{~nm}$ spectral line produced by the $(3,1)$ to $(2,0)$ atomic transition and the $\lambda_{2}=$ $656.4695 \mathrm{~nm}$ spectral line, which was estimated as in the middle of the spectral 
Table 2. Experimental (second column) and calculated (third column) wavelengths corresponding to the doublet structure of the Hydrogen's Lyman (first two rows) and Balmer $\alpha$-lines (last two rows).

\begin{tabular}{ccc}
\hline$(\mathrm{In} \mathrm{nm})$ & Experimental & Calculated \\
\hline$\alpha$-Lyman $(\lambda$ in nm $)$ & 121.567 & 121.558 \\
$\alpha$-Lyman $(\Delta \lambda$ in nm $)$ & 0.006 & No \\
$\alpha$-Balmer $(\lambda$ in nm $)$ & 656.279 & 656.422 \\
$\alpha$-Lyman $(\Delta \lambda$ in meV $)$ & 0.04 & 0.16 \\
\hline
\end{tabular}

lines corresponding to the atomic transitions $(3,0)$ to $(2,1)$ and $(3,2)$ to $(2,1)$. This corresponds to a Balmer's $\alpha$-doublet separation of $\Delta \lambda \sim 0.048 \mathrm{~nm}$ or $\Delta E \sim$ $0.16 \mathrm{meV}$. Nevertheless, as shown in Table 2, this value is four times larger than the experimental value [14], which demonstrates the need for including in the calculation both the Darwin and the spin-orbit contributions.

\section{Conclusion}

It has been shown how to solve the Grave de Peralta equation for a charged quantum particle with mass and spin- 0 , which is moving in a Coulomb potential or contained in a spherical infinite well. The solutions were found following the same procedures and with no more difficulty than the corresponding to solving the same problems using the Schrödinger equation. Nevertheless, the solutions found in this work are also valid when the particle is moving with quasi-relativistic energies. For instance, it was shown that the energies of the electron in a Hydrogen atom, which were calculated by solving the Grave de Peralta equation, includes the relativistic Thomas correction. Moreover, the relativistic correction to the kinetic energy is just an approximation found using a perturbative approach while Equation (63) was exactly solved. In addition, it should be noted that Equation (41) is different than the radial equation obtained using the Schrödinger equation. The author is currently working on solving Equation (41). This will allow to obtain more precise expressions for the atomic orbitals currently used in numerous $a b$ initio computer packages dedicated to computer calculations in physical-chemistry and atomic and solid-state physics.

\section{Conflicts of Interest}

The author declares no conflicts of interest regarding the publication of this paper.

\section{References}

[1] Bohm, D. (1964) Quantum Theory. 11th Edition, Prentice-Hall, USA.

[2] Davydov, A.S. (1965) Quantum Mechanics. Pergamon Press, Oxford.

[3] Merzbacher, E. (1970) Quantum Mechanics. 2nd Edition, John Wiley \& Sons, New York.

[4] Griffiths, D.J. (1995) Introduction to Quantum Mechanics. Prentice Hall, USA. 
[5] Levine, I.N. (2014) Quantum Chemistry. 7th Edition, Pearson Education, New York.

[6] Home, D. (1997) Conceptual Foundations of Quantum Physics: An Overview from Modern Perspectives. Plenum Press, New York.

[7] Grave de Peralta, L. (2020) Journal of Modern Physics, 11, 196-213. https://doi.org/10.4236/jmp.2020.112012

[8] Strange, P. (1998) Relativistic Quantum Mechanics: With Applications in Condensed Matter and Atomic Physics. Cambridge University Press, New York. https://doi.org/10.1017/CBO9780511622755

[9] Greiner, W. (1990) Relativistic Quantum Mechanics: Wave Equations. Spring-Verlag, New York.

[10] Jackson, J.D. (1975) Classical Electrodynamics. 2nd Edition. John Wiley \& Sons, New York.

[11] Grave de Peralta, L. (2020) Results in Physics, Under Review.

[12] Nanni, L. (2015) arXiv:1501.05894.

[13] Parthey, C.G. (2011) Ludwig Maximilian University of Munich. http://citeseerx.ist.psu.edu/viewdoc/summary?doi=10.1.1.232.5350

[14] Lewis, G.N. and Spedding, F.H. (1933) Physical Review, 43, 964. https://doi.org/10.1103/PhysRev.43.964 